\title{
Role of natural killer cells for immunotherapy in chronic myeloid leukemia (Review)
}

\author{
HYE-RIM LEE and KWANG-HYUN BAEK \\ Department of Biomedical Science, CHA University, CHA General Hospital, \\ Seongnam, Gyeonggi 13488, Republic of Korea
}

Received January 8, 2019; Accepted February 6, 2019

DOI: $10.3892 /$ or.2019.7059

\begin{abstract}
The majority of natural killer (NK) cells serve an important role in eliminating malignant cells. The cytotoxic effects of NK cells were first identified against leukemia cells, and it is now hypothesized that they may have a critical role in leukemia therapy. The cellular functions of NK cells are mediated by their cell surface receptors, which recognize ligands on cancer cells. The role of NK cells is specifically regulated by the activating or inhibitory killer cell immunoglobulin-like receptors (KIRs) on their surface, which bind to the human leukocyte antigen (HLA) class I ligands present on the target cells. The association between KIR and HLA is derived from the diversity of $K I R / H L A$ gene profiles present in different individuals, and this determines the cytotoxic effect of $\mathrm{NK}$ cells on cancer cells. Chronic myeloid leukemia (CML) is a hematological leukemia characterized by the hyper-proliferation of myeloid cells, with the majority of patients with CML presenting with abnormal immune cells. Tyrosine kinase inhibitors are the present standard therapy for CML, but are associated with numerous adverse side effects. Various studies have proposed CML therapy by immunotherapeutic approaches targeting the immune cells. This review summarizes the contents of $\mathrm{NK}$ cells and the association between KIR/HLA and leukemia, especially CML. This is followed by a discussion on the development of NK cell immunotherapy in hematological malignancies and research into strategies to enhance NK cell function for CML treatment.
\end{abstract}

Correspondence to: Professor Kwang-Hyun Baek, Department of Biomedical Science, CHA University, CHA General Hospital, 335 Pangyo-Ro, Bundang-Gu, Seongnam, Gyeonggi 13488, Republic of Korea

E-mail: baek@cha.ac.kr

Key words: natural killer cell receptors, killer cell immunoglobulinlike receptor-human leukocyte antigen mismatch, natural killer cell signaling, chronic myeloid leukemia therapy

\section{Contents}

1. Natural killer (NK) cells and their markers

2. Types of NK cell receptors

3. Fundamentals of NK cell alloreactivity (inhibitory receptors)

4. Fundamentals of NK cell alloreactivity (activating receptors)

5. KIRs

6. Models to determine NK cell KIR alloreactivity for cell therapy

7. Mechanism of NK cell cytotoxicity in cancer

8. Types of leukemia and the therapeutic effect of NK cells

9. Therapeutic targets in CML

10. Therapeutic targets in CML by immunotherapy

11. Therapeutic targets in CML by NK cells

12. NK cell alloreactivity controls the GVH effect

13. Adoptive NK cell therapy in hematopoiesis

14. KIR genes in patients with leukemia

15. Conclusions

\section{Natural killer (NK) cells and their markers}

NK cells are innate lymphoid immune cells comprising $5-20 \%$ of the peripheral blood, and serve an important role in the immune control of cancer $(1,2)$. They are identified by the expression of the surface markers cluster of differentiation (CD) 56 and CD16, and the lack of CD3; the CD3 subset therefore indicates T-cell depletion. The two major NK cell subset markers, CD56 and CD16, are present in human cells, and induce different functions in NK cells. The $\mathrm{CD} 56^{\text {bright }} \mathrm{CD} 16^{-}$ subset has specialized proliferative potential, whereas $\mathrm{CD} 56^{\mathrm{dim}} \mathrm{CD} 16^{+}$facilitates the cytolytic activity of NK cells. The higher density of lytic granules in CD56 $6^{\mathrm{dim}}$ cells and the capacity to trigger cytokine production of $\mathrm{CD}^{+} 6^{+}$cells confers cytotoxic activity on NK cells (1-3) (Fig. 1).

\section{Types of NK cell receptors}

NK cell activity is adapted by a number of gene families encoding inhibitory and activating receptors. The major histocompatibility complex (MHC) class I-binding or non-binding receptors include two principal types of receptors. The NK cell receptor families belonging to MHC class I-binding receptors are the c-type lectin receptors (CLRs), apart from 
NKG2-D type II integral membrane protein (NKG2D), leukocyte immunoglobulin-like receptors (LIRs) and killer cell immunoglobulin-like receptors (KIRs). On the other hand, natural cytotoxicity receptors (NCRs) and other NK cell receptors recognize tumor cells independently of MHC class I molecules (1) (Fig. 2).

The CLRs of NK cell receptors structurally contain the c-type lectin domain in their extracellular region. Lectins contain a carbohydrate-binding protein domain, and the c-type form requires calcium in order to bind with proteins. These lectins are diversely functional in their immunopathological reactions in the context of NK cell interactions.

The LIRs are a multigene family located on chromosome 19 , including the KIR gene family, containing Ig-like extracellular domains in their cytoplasmic tails. Even though LIRs bind to MHC class I molecules, they do not have an important role in NK cell interactions since the binding of LIR to UL18 has a higher affinity compared with the binding of LIR-1 to MHC class I. The LIR uses the same binding site to interact with MHC class I molecules or UL18, an example of which being the human cytomegalovirus genes (1,4-6).

There are two types of KIRs: The inhibitory KIR (iKIR) and activating KIR (aKIR). Structurally, KIRs have two or three immunoglobulin-like domains that are transmembrane glycoproteins. The cytoplasmic tail of iKIR contains the immunoreceptor tyrosine-based inhibitory motif (ITIM), whereas the short cytoplasmic tails of aKIR contain the immunoreceptor tyrosine-based activation motif (ITAM) and have a positively charged residue.

$\mathrm{CD} 56^{\mathrm{dim}} \mathrm{CD} 16^{+} \mathrm{NK}$ cells express high levels of KIRs and CLRs compared with CD56 ${ }^{\text {bright }} \mathrm{CD} 16^{-} \mathrm{NK}$ cells. Furthermore, $\mathrm{CD} 56^{\mathrm{dim}} \mathrm{CD} 16^{+} \mathrm{NK}$ cells have a higher affinity and recognize target cells bearing MHC class I ligands, thereby exerting greater cytotoxicity compared with $\mathrm{CD} 56^{\text {bright }} \mathrm{CD} 16^{-} \mathrm{NK}$ cells. Therefore, this review focuses on the KIRs of NK cell receptors, due to their increased recognition and interaction with MHC class I molecules on target cells (4).

The NCRs and other NK cell receptors recognize tumor cells irrespective of MHC class I. NCRs are of three types: NKp46, NKp44 and NKp30. NKp30 have a crystal and dimeric structure, NKp44 comprises a typical v-type Ig-fold, whereas NKp46 consists of labeled $\beta$ strands. However, at present, the NCRs and their ligand interactions are poorly understood $(5,7)$.

\section{Fundamentals of NK cell alloreactivity (inhibitory receptors)}

Since NK cells spontaneously circulate throughout the human body via the peripheral blood, it is essential to have inhibitory receptors that impede their attack on healthy tissues. The majority of inhibitory receptors recognize the MHC class I molecules present on the surface of cells. Since MHC class I molecules are downregulated in tumor cells or virus-infected cells (unhealthy cells), the NK cells are able to selectively eliminate these unhealthy cells via a process termed 'missing-self' (8) (Fig. 3). CD94/NKG2-A/NKG2-B type II integral membrane protein, the principal inhibitory receptor of NK cells, which binds to the MHC class I molecule HLA-E, is present on target cells (9). The other inhibitory receptors are primarily iKIRs, which bind to the MHC class I molecules HLA-A, B and C $(9,10)$. Since NK cell receptors are involved in the binding of ligands, it is important to establish strategies that kill tumor cells via NK cell cytotoxicity.

Inhibitory NK cell receptors exert their effect by obstructing the activation of NK cells. The inhibitory NK cell receptors generally express the ITIM, which recruits and phosphorylates Src homology (SH)-containing tyrosine phosphatase (SHP)-1 and SHP-2. Inhibition by ITIM-bearing receptors hampers the tyrosine phosphorylation of essential signaling components, including tyrosine phosphoprotein SLP-76 (SLP-76) and proto-oncogene vav (Vav), to activate signaling in NK cells. However, non-MHC-binding inhibitory receptor mechanisms have various signaling pathways (11-14) (Fig. 4). Specifically, 2B4 of NK cell receptors bind to CD48, which exists on all human hematopoietic cells. 2B4 comprises four tyrosine motifs (TxYxxI/V) in the cytoplasmic tail, and the immunoreceptor tyrosine-based switch motif interacts with SLAM-associated protein. The function of 2B4 has been demonstrated to involve the recruitment of signaling components of other activating receptors (12).

\section{Fundamentals of NK cell alloreactivity (activating receptors)}

The activation of NK cells requires external signals via their activating receptors that bind to the ligands on target cells. Other important activating receptors include the NKG2 members (excluding $\mathrm{A}$ and $\mathrm{B}$ ), NCRs and aKIRs. NKG2C, $\mathrm{E}$ and $\mathrm{H}$ bind to the MHC class I molecule HLA-E, whereas NKG2D binds to MHC class I chain-related genes A and B (MICA and MICB) and UL-16 protein-ligand family 1 and 6 (ULBP1 and ULBP6). aKIR binds to the MHC class I molecules HLA-C or G, and another unknown receptor $(3,14-16)$.

The activating NK receptors possess small transmembrane-anchored adaptor proteins that express ITAM in addition to ITAM-bearing polypeptides (CD3 $\xi$, FcR $\gamma$, DAP10 and DAP12) in their intracellular membrane. ITAM-bearing receptor complexes facilitate the recruitment of tyrosine-protein kinase SYK (Syk) and tyrosine-protein kinase ZAP-70 (ZAP70). Moreover, since CD3५ and FcR $\gamma$ facilitate CD16 signaling in NK cells, the ITAM-bearing receptor complexes phosphorylate src family kinases. The recruitment and phosphorylation of Syk and ZAP70 promote the signals that trigger the activation of SLP-76, SHC-transforming protein 1 (Shc), linker for activation of T-cells family member 1 (LAT), growth factor receptor-bound protein 2 and SH3 domain-binding protein 2, which are linkers for the activation of proto-oncogene Vav and Ras-related C3 botulinum toxin substrate 1 . These signals produce downstream events, namely the activation of the mitogen-activated protein kinase (MAPK) and extracellular signal-regulated kinase (ERK) pathway (11-14) (Fig. 4).

One example is the NCRs, comprising three major proteins (NKp46, NKp30 and NKp44) that have an adaptor protein, ITAM. Activation of the NCRs is associated with ITAM-bearing receptor complexes that induce signaling pathways such as MAPK and ERK. Other examples are the NKG2 members, which are associated with TYRO protein tyrosine kinase-binding protein/DNAX-activation protein (DAP) 10 or 


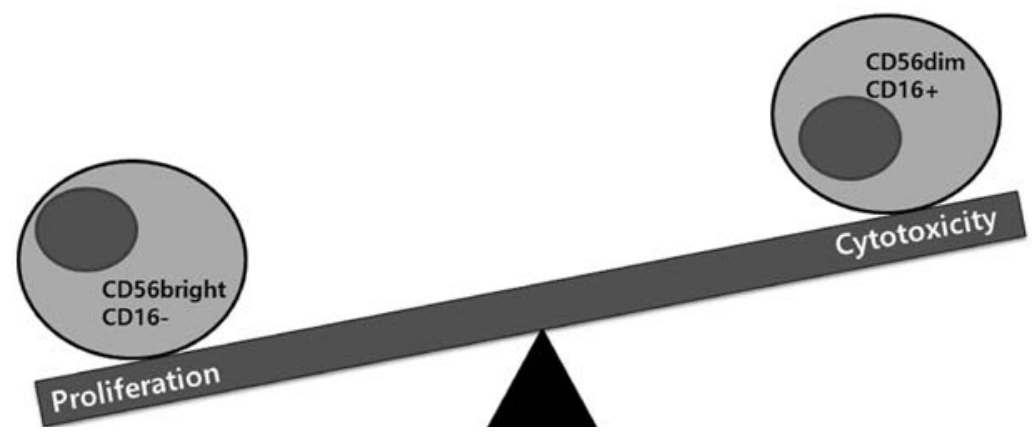

Figure 1. Functions of human $\mathrm{CD} 56^{\text {bright }} \mathrm{CD} 16^{-}$and $\mathrm{CD} 56^{\mathrm{dim}} \mathrm{CD} 16^{+} \mathrm{NK}$ cells. The principal NK cell markers that induce different functions in $\mathrm{NK}$ cells are $\mathrm{CD} 56$ and $\mathrm{CD} 16$. The $\mathrm{CD} 56^{\text {bright }} \mathrm{CD} 16^{-}$subset has proliferative potential, whereas the $\mathrm{CD} 56^{\mathrm{dim}} \mathrm{CD} 16^{+}$subset possesses cytolytic activity. $\mathrm{CD}$, cluster of differentiation; NK, natural killer.

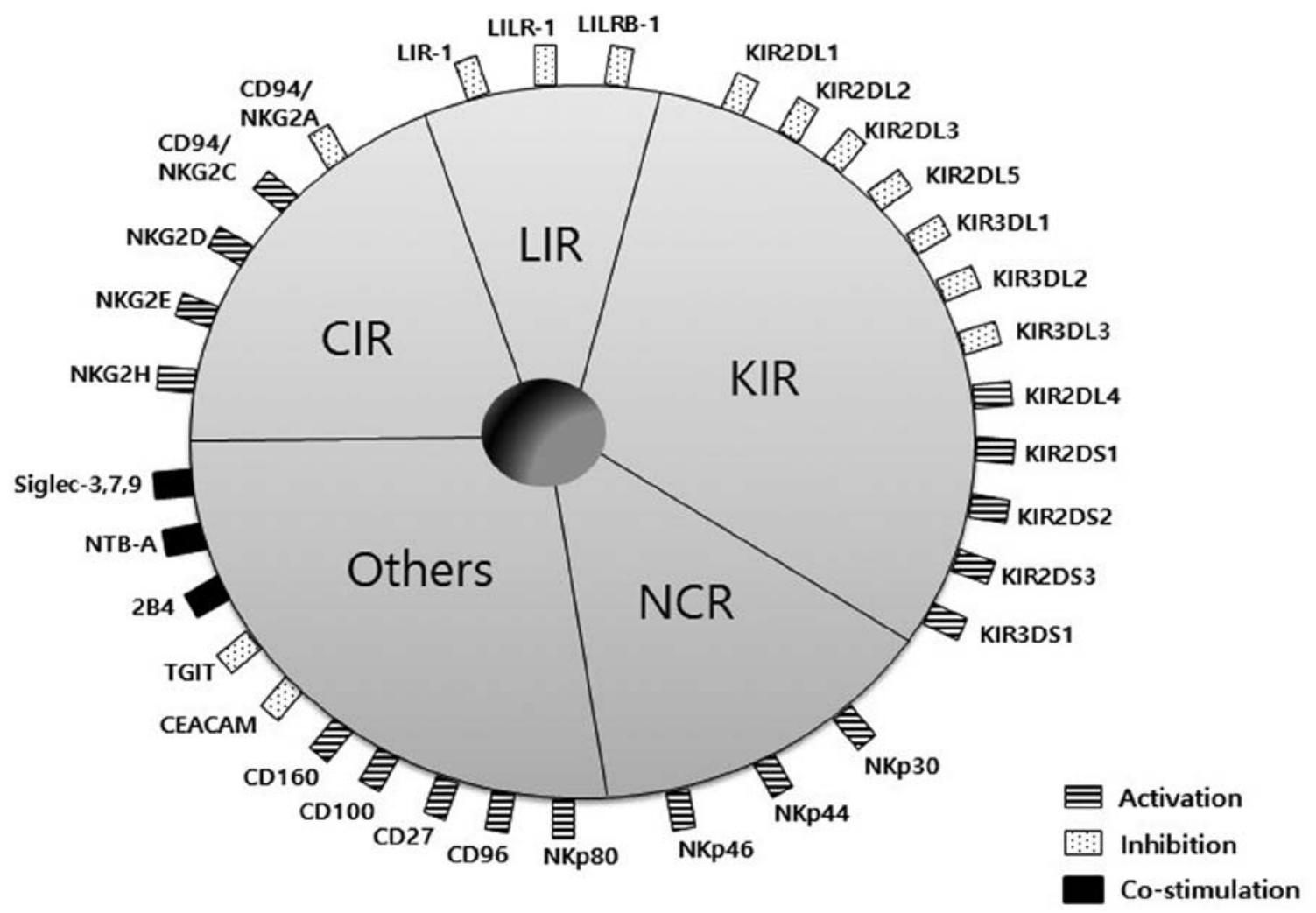

Figure 2. NK cell receptors. The different types of NK cell receptors are presented: CLRs, LIRs, KIRs, NCRs and others. CLR, c-type lectin receptor; LIR, leukocyte immunoglobulin-like receptor; KIR, killer cell immunoglobulin-like receptor; NCR, natural cytotoxicity receptor; CD, cluster of differentiation; NK, natural killer.

DAP12 adaptor proteins. In particular, NKG2D is expressed on the NK cell surface and binds to its ligands on target cells (MICA, MICB and ULBP) in humans, resulting in the expression of DAP10 and DAP12. NKG2D is further classified as the short form (NKG2D-S) or the long form (NKG2D-L), in line with the size of its cytoplasmic tail. NKG2D-S binds to either DAP10 or DAP12, but NKG-L is able to bind only to DAP10. In humans, NKG2D pairs only with DAP10 subsequent to the stimulation of the p85 PI3 kinase-associated pathway. In addition, the NKG2D signaling pathway has cross-linking signals that are associated with the activation of the signal transducer and activator of transcription (STAT)5, tyrosine-protein kinase JAK2 (JAK2), ERK, MAPK and phosphatidylinositol 3-kinase/RAC- $\alpha$ serine/threonine-protein kinase signal transduction pathways $(11,13)$.

\section{KIRs}

Due to their individual diversity, KIRs serve a pivotal role in human NK cells with respect to targeting cells. In terms of gene content, KIR receptors are classified as iKIR (2DL1, 2DL2, 2DL3, 3DL1, 3DL2, 3DL3, and 2DL5A and B) and aKIR (2DL4, 2DS1, 2DS2, 2DS3, 2DS4, 2DS5 and 3DS1). The $K I R$ gene content defines the KIR haplotype of an individual. KIR haplotypes with distinct gene content are distinguished as 'A haplotype' and 'B haplotype'. The A haplotype exists in all populations conventionally, and has a fixed gene content of 9 genes (3DL3, 2DL3, 2DP1, 2DL1, 3DP1, 2DL4, 3DL1, 2DS4 and 3DL2). Different B haplotypes exist among different people (2DS1, 2DS2, 2DS3, 2DS5, 2DL2, 2DL1 and 3DS1). It encodes more active KIRs compared with the A 


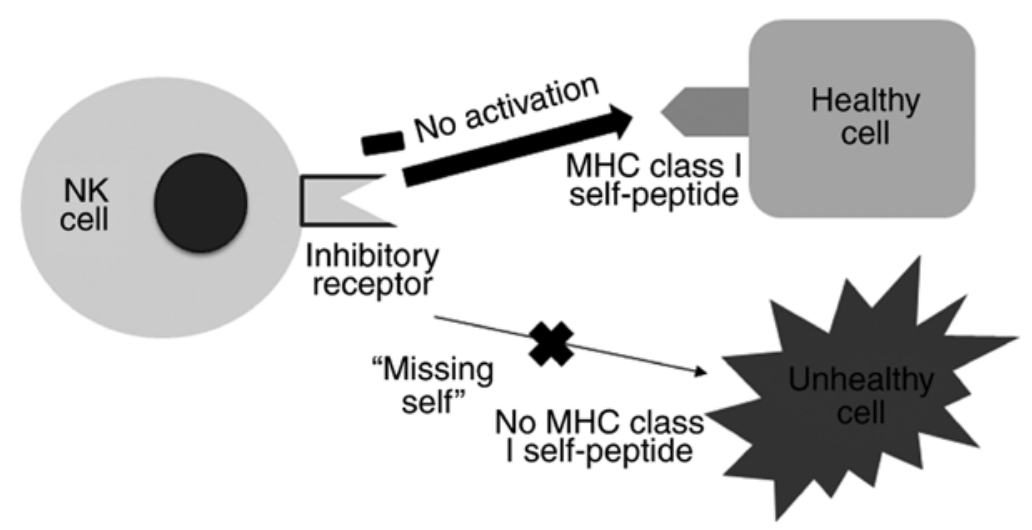

Figure 3. Concept of 'missing-self' with respect to NK cells. The 'missing-self' concept is a professional killer cell response, allowing NK cells selectively remove only unhealthy cells. NK, natural killer; MHC, major histocompatibility complex.

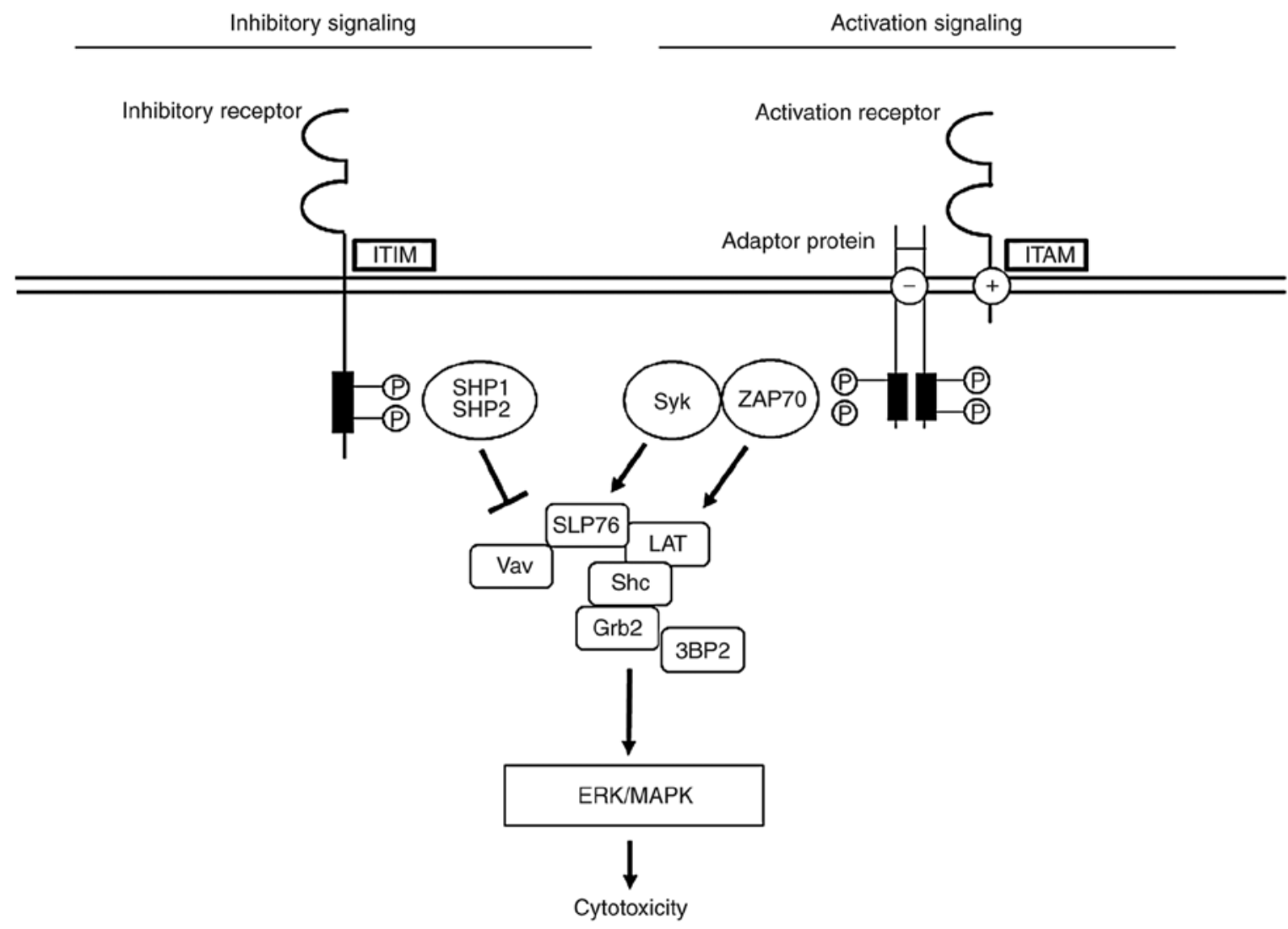

Figure 4. NK cell receptor signaling pathways associated with ITIM- or ITAM-containing adaptor proteins. In these models, the phosphorylation of ITIM leads to the recruitment of SHP-1 and SHP-2, which disturb SLP76 and Vav and are associated with activating signals. On the other hand, the phosphorylation of adaptor proteins with an ITAM triggers the recruitment of Sky and ZAP-70, which activate various signaling molecules, including SLP-76, LAT, Shc, $\mathrm{Gb} 2$ and 3BP2. Signals for cytotoxicity result in the activation of downstream activating pathways, via the ERK/MARK pathway. ITIM, immunoreceptor tyrosine-based inhibitory motif; ITAM, immunoreceptor tyrosine-based activation motif; NK, natural killer; SHP, Src homology-containing tyrosine phosphatase; SLP-76, tyrosine phosphoprotein SLP-76; Vav, proto-oncogene vav; Syk, tyrosine-protein kinase SYK; ZAP70, tyrosine-protein kinase ZAP-70; LAT, linker for activation of T-cells family member 1; Shc, SHC-transforming protein 1; Grb2, growth factor receptor-bound protein 2; 3BP2, SH3 domain-binding protein 2; MAPK, mitogen-activated protein kinase; ERK, extracellular signal-regulated kinase.

haplotype $(1,17,18)$. Specifically, iKIRs have a long tail in their intracytoplasmic region, denoted by the letter 'L'; aKIRs have comparatively shorter tails, indicated by the letter 'S'. Also, KIR polymorphism is characterized by a different structure, wherein the iKIR contains the ITIM in the cytoplasmic tails, and aKIRs contain positively charged amino acid residues in the transmembrane regions (19).
Although they recognize identical MHC class I alleles, the inherent differences between iKIR and aKIR lead to different outcomes, namely inhibitory or activating responses, respectively. As previously described in this review, iKIR signaling results from the existence of ITIMs that induce inhibitory signaling in connection with SHP-1 and SHP-2. Conversely, aKIR signaling involves ITAMs and may produce 


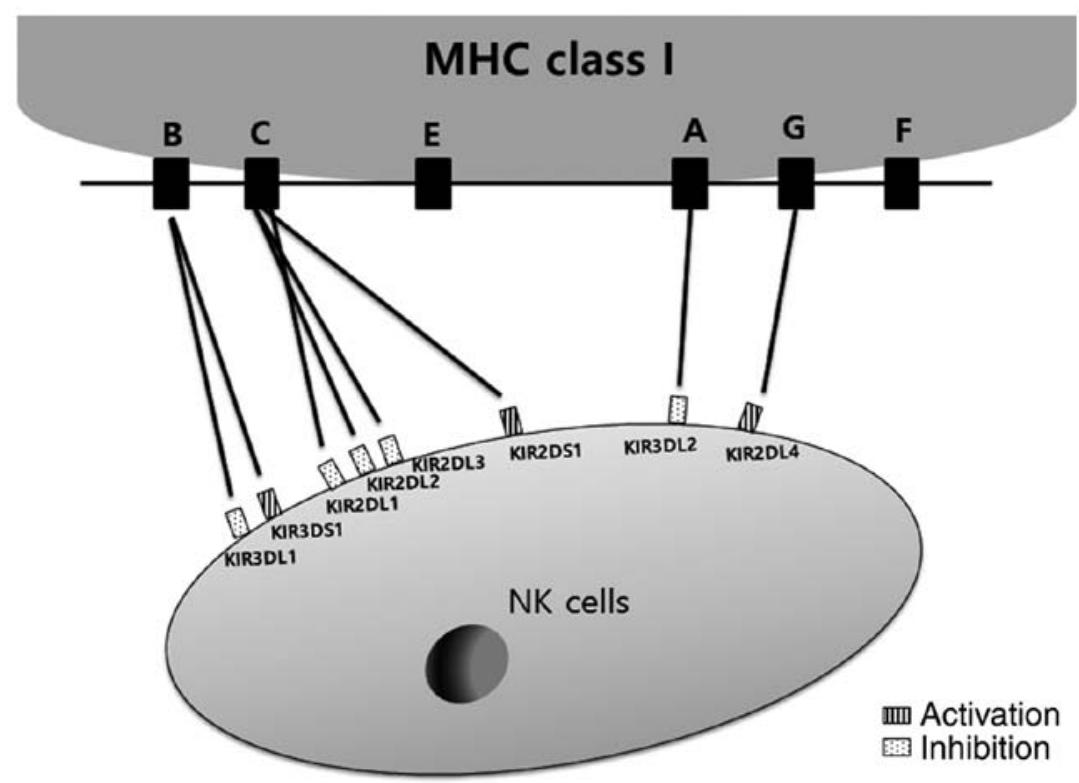

Figure 5. Binding between KIR and specific ligands of MHC class I subunit. Since NK cell receptors have ligand specificity, the NK cell repertoire with diverse combinations of KIRs required further research to investigate matching or mismatching between KIRs and KIR ligands. MHC, major histocompatibility complex; NK, natural killer; KIR, killer cell immunoglobulin-like receptor.

ITAM-bearing receptor complexes that activate signaling molecules, including Syk, ZAP70, SLP-76, Shc and LAT, resulting from the activation of downstream signals, including the MAPK and ERK pathway (4,19) (Fig. 4).

\section{Models to determine NK cell KIR alloreactivity for cell therapy}

Functional capacity is induced by the interaction between KIR receptors and specific KIR ligands. Inhibitory KIR receptors predominantly bind to distinct $\mathrm{MHC}$ class I molecule HLA subtypes, but the ligands for activating KIR receptors are unknown. It has been identified that the activating KIR2DS1 recognizes MHC class I molecule HLA-C subtype ligands $(1,18)$. Thus, the repertoire of NK cells with diverse combinations of KIRs requires more detailed study to investigate KIR-KIR ligand matches or mismatches due to their requirement for type specificity $(3,17)$ (Fig. 5). Notably, an A haplotype KIR-KIRL mismatched donor-recipient has been described in allogenic hematopoietic transplantation for the treatment of leukemia (20). The process of 'licensing' of NK cells may be beneficial as a graft-versus-host $(\mathrm{GVH})$ effect. This means that the iKIR of licensed NK cells is not recognized by KIR ligands, and the absence of MHC class I molecule HLA subset expression on the leukemia cells of the recipient is able to trigger NK cell alloreactivity. Donor NK cell alloreactivity has a critical role in the GVH effect compared with donor $\mathrm{T}$ cell allografts, since the alloreactivity of $\mathrm{T}$ cells may negatively affect healthy recipient cells $(18,21)$.

\section{Mechanism of NK cell cytotoxicity in cancer}

NK cells release various cytokines and acquire certain cytotoxic effects. NK cells recognize tumor cells by their surface markers or receptors in order to activate NK functions via either of the two tumor-recognizing models: 'Missing-self recognition' and 'stress-induced recognition'. The missing-self recognition model encompasses a loss of the inhibitory effect of NK cells resulting from the absence of MHC class I molecules on tumor cells. In the stress-induced recognition model, damaged proteins on tumors bind to the activating receptors of NK cells and stimulate the cytotoxic functions of NK cells (22-24).

NK cell activation facilitates killing of tumor cells either through direct or indirect means. In the first mechanism, granules are released, including perforin and granzymes, which induce apoptosis in tumor cells $(22,25)$. The second mechanism includes antibody-dependent cell-mediated cytotoxicity, wherein the Fc region of the IgG antibody binds to antigens on the tumor cells in addition to the CD16 receptor on NK cells, forming cross-linking via CD16. The cross-linking of CD16 promotes the granule-dependent mechanism that kills the tumor cells via the activated NK cells by apoptosis $(22,26,27)$. A third mechanism is death receptor-dependent apoptosis. This mechanism involves the Fas ligand and tumor necrosis factor (TNF)-related apoptosis-inducing ligand, which induce caspase-dependent apoptosis in tumor cells $(22,25,28)$. An indirect model of activated $\mathrm{NK}$ cells involves the secretion of cytokines and chemokines, including interferon (IFN)- $\alpha$, TNF- $\alpha$, C-C motif chemokine (CCL)3, CCL4 and CCL5. A number of cytokines enhance the proliferation and activation of immune cells, such as monocytes, macrophages, dendritic cells (DCs), B-cells and cytotoxic T lymphocytes (CTLs). These cells are cytotoxic towards tumor cells, which die due to the immune response associated with apoptosis and necrosis $(22,25,26,29)$. Further studies are required to focus on NK cell immunotherapy for cancer, since NK cells serve an important role in the immune response and are capable of killing cancer cells. 
Table I. Expression of ligands and receptors in patients with hematological cancer.

Expression levels in patients

\begin{tabular}{|c|c|c|c|c|}
\hline & & & & \\
\hline Author, year & Type of cancer & Ligands & Receptors & (Refs.) \\
\hline Nückel et al, 2010 & CLL & $\begin{array}{l}\mathrm{MICA} / \mathrm{B} \text {, absent; } \\
\text { ULBP, absent }\end{array}$ & - & (30) \\
\hline Osman et al, 2017 & ALL & $\begin{array}{l}\text { MICA/B, downregulated; } \\
\text { ULBP, downregulated }\end{array}$ & - & (32) \\
\hline Osman et al, 2017 & AML & DNAM-1, downregulated & $\begin{array}{l}\text { NCR, } \\
\text { downregulated; } \\
\text { NKG2D, downregulated; } \\
\text { CD94/NKG2C, } \\
\text { downregulated }\end{array}$ & (32) \\
\hline Boissel et al, 2006 & CML & MICA, upregulated & NKG2D, downregulated & (33) \\
\hline
\end{tabular}

CLL, chronic lymphocytic leukemia; ALL, acute lymphocytic leukemia; AML, acute myelogenous leukemia; CML, chronic myelogenous leukemia; MIC, MHC class I chain-related gene; ULBP, UL-16 protein-ligand family; DNAM-1, CD 226 antigen; NCR, natural cytotoxicity receptor; NKG2D, NKG2-D type II integral membrane protein; CD, cluster of differentiation.

\section{Types of leukemia and the therapeutic effect of NK cells}

Among hematological cancers, leukemia exhibits rapid proliferation of abnormal white blood cells in the bone marrow. Leukemias are classified into four major types: Acute lymphocytic leukemia (ALL), acute myelogenous leukemia (AML), chronic lymphocytic leukemia (CLL) and chronic myelogenous leukemia (CML). It is important to understand the various leukemia types, since each type requires a different treatment. In leukemia, NK cells exert their potential therapeutic effect by eliminating abnormal leukemic cells due to their cytotoxicity. Various therapeutic options for leukemia have been associated with NK cell receptors (16).

ALL, a cancer of the lymphoid cells characterized by an increased number of lymphocytes, is the most common childhood leukemia. Although ALL is resistant to NK cell cytotoxicity, recent clinical studies have revealed the therapeutic effect of NK cells in pediatric patients $(1,17)$. CLL encompasses the B-cell subtype of lymphocytes (B-CLL) in the bone marrow. Since CLL blasts do not express the MICA, MICB and ULBP ligands, NK cells are capable of eradicating these blasts (30-32). CML involves the constant proliferation of myeloid cells, particularly mature granulocytes, which are clonal bone marrow stem cells. Thus, CML is referred to as a clonal disorder, since the majority of patients with CML bear the distinctive feature of a chromosomal translocation termed Philadelphia chromosome $(\mathrm{Ph}) . \mathrm{Ph}$ is a mutual translocation between the 9th and 22nd chromosomes in all hematopoietic cells; the Abelson ( $a b l)$ gene on chromosome 9 is transferred to the breakpoint cluster region ( $b c r$ ) gene from chromosome 22. This translocation results in an abnormal 'fusion' termed the $b c r / a b l$ gene. The $b c r / a b l$ gene develops a tyrosine kinase protein, since the $a b l$ gene product adds phosphate groups to tyrosine residues $(33,34)$. As a result, CML is a clonal myeloproliferative disease of abnormal translocation of the $b c r-a b l$ oncogene, which produces the oncokinase protein BCR-ABL, responsible for the pathogenesis of CML $(34,35)$.
In the search for CML therapies, previous in vitro experiments investigated NK-92MI cell line cytotoxicity against the K562 leukemia cell line $(36,37)$. In addition, CML may be treated with tyrosine kinase inhibitors (TKIs) due to the presence of high levels of MICA and low expression of NKG2D $(33,36)$. Therefore, for the effective treatment of cancer using immunotherapy, such as NK cells, it is important to understand the expression of ligands and receptors in patients with hematological cancer, which are summarized in Table I. This review particularly focuses on anti-leukemic effects in CML. Notably, NK cells exert their cytotoxic effect by eliminating CML leukemic cells through lysis.

\section{Therapeutic targets in CML}

As previously discussed in this review, the $b c r-a b l$ oncogene is a critical gene in CML $(34,35)$. Thus, novel strategies targeting CML use TKIs, including imatinib mesylate, which provide improved long-term survival of patients (38). In the majority of early studies, researchers proposed that TKIs could be an important therapeutic avenue for CML treatment, although there was limited information about the underlying molecular mechanism. To be specific, statistics indicate that imatinib mesylate is one of the TKIs that induces a complete hematological and cytogenetic response in $\sim 83 \%$ of CML patients for 10 years $(37,38)$. However, certain patients with CML are resistant to the therapy due to mutations in the ABL kinase domain; furthermore, the treatment is associated with long-lasting side effects including nausea, cramps and peripheral edema. In addition, TKI therapy involves a high drug cost $(39,40)$. Second-generation TKIs, including dasatinib and nilotinib are under investigation for the treatment of CML. To date, the number of patients with CML who have benefitted from TKI treatment remains unknown (41-44). Therefore, the development of new TKI agents and combination therapies with other treatment strategies is urgently required for TKI therapy. If certain patients with CML exhibit resistance to 
Table II. Immunotherapeutic approaches for CML.

\begin{tabular}{lll}
\hline Author, year & Pathway or target & Treatment strategy \\
\hline
\end{tabular}

Cayssials and Guilhot, 2017; $\quad$ PD-1+ cytotoxic T cells
Mahoney et al, 2015

Cayssials and Guilhot, 2017; $\quad$ PD-L1+ myeloid leukemic cells $\quad$ PD-1/PD-L1 antagonists

Mahoney et al, 2015;

Mumprecht et al, 2009

Zhang et al, 2016;

Stramucci and Perrotti, 2016;

Ågerstam et al, 2016;

Arranz et al, 2017;

Zhao et al, 2017

Tarafdar et al, 2017;

Levescot et al, 2014;

Rocca et al, 2018

IL-1 signaling in CML stem cells Monoclonal antibodies CAR-T cells

CML, chronic myelogenous leukemia; PD-1, programmed cell death protein 1; PD-L1, PD-1 ligand 1; JAK, tyrosine-protein kinase JAK; STAT, signal transducer and activator of transcription; CAR-T, specific chimeric antigen receptor-modified T; IL-1, interleukin-1.

TKIs, other approaches are required to improve the treatment of CML. A number of patients with CML have exhibited a complete cytogenetic response with no recurrence within 10 years following IFN- $\alpha$ treatment (45). IFN- $\alpha$ inhibits cell proliferation, regardless of the $b c r-a b l$ oncogene. There remain certain problems associated with this treatment, since certain CML patients receiving IFN- $\alpha$ therapy experience side effects and develop tolerance to IFN- $\alpha(39,45)$.

Treatment with hydroxyurea, leukapheresis and stem cell transplantation are other options in the treatment of CML. Hydroxyurea is generally administered prior to treatment with imatinib mesylate. Nevertheless, hydroxyurea has demonstrated efficacy in patients with CML of up to $90 \%$, but is not commonly utilized in CML therapy for long-term administration due to its inherent cytotoxicity (46-48). Leukapheresis is another treatment applied to reduce the high levels of white blood cells in patients with CML, and stem cell transplantation has also been considered as another treatment option $(49,50)$.

Considering all of the above, developing novel effective treatment strategies for patients with CML is a clear medical necessity. The understanding of tumor immunology has allowed for considerable progress, which has been applied to the immunotherapy of various tumors. Since immunosurveillance is impaired in patients with CML, the lack of innate immune cells including NK cells, B-cells, T-cells and dendritic cells results in decreased quantitative and qualitative functions in the blood (51).

\section{Therapeutic targets in CML by immunotherapy}

Previous studies have included various immunotherapeutic approaches for CML by targeting the underlying pathways, such as the programmed cell death protein 1 (PD-1)/PD-1 ligand 1 (PDL-1), interleukin (IL)-1 and JAK/STAT pathways (47-52) (Table II).
The PD-1/PDL-1 interaction may act as an immune checkpoint inhibitor, having a marked effect on the anti-tumor response. To facilitate signaling by PD-1, binding of PDL-1 is essential. PD-1 signaling may be involved in the functional effect of infiltrating $\mathrm{T}$ cells in tumors. In patients with CML, PD-1 is highly expressed on CML-specific CTLs, whereas the CML cells express PDL-1. Thus, PD-1/PDL-1 interaction leads to the exhaustion and inhibition of CTLs in patients with CML (53). Therefore, blocking PD-1/PDL-1 binding as a treatment strategy represents a promising therapeutic approach for CML (51-53). IL-1 is a regulator of inflammation and the innate immune response, having numerous roles in immunopathological functions. In hematological malignancies, drugs that target IL-1 have an important therapeutic value. Recently, there have been numerous clinical trials in patients focusing on treatments targeting the IL-1 signaling pathway $(54,55)$. In CML, since IL-1 provides resistance to imatinib in CML, the prevention of IL-1 signaling has the potential to increase the efficacy of TKI treatment. Additionally, when using IFN- $\alpha$ treatment in CML, IFN- $\alpha$ inhibits IL-1 due to its anti-inflammatory effect. Hence, a combination therapy with TKIs and IFN- $\alpha$ may be a more efficacious approach for CML patients. Meanwhile, IFN- $\alpha$-resistant CML patients have high levels of IL-1, which stimulates IFN- $\alpha$-sensitive CML cells. One way of the blocking IL-1 pathway is using IL-1 receptor accessory (IL1RAP)-specific chimeric antigen receptor-modified T (CAR-T) cells. IL1RAP-CAR-T cells represent an important alternative therapy for patients with CML presenting with TKI and IFN- $\alpha$ resistance (56-58).

The tyrosine kinase JNK has a critical role in myelopoiesis by transducing cytokine signals, and binds to the cytoplasmic domains of various cytokines and growth factor receptors. Once the binding of ligands to their receptors in the extracellular region occurs, JAK is phosphorylated in the intracellular region. Phosphorylated JAK activates downstream substrates 
such as the intracellular transcription factor STAT. The JAK-STAT pathway is involved in facilitating myeloproliferation and is abnormally activated in hematopoietic diseases $(59,60)$. Therefore, JAT-STAT inhibitors merit consideration for the treatment of CML. Furthermore, patients with JAK-mutated CML may benefit from TKI treatment, since the JAK-STAT pathway is not normally activated. Thus, randomized trials are required to confirm the potential advantages of the therapy in this cohort (61).

As a novel therapy for CML, NK cells have potential for use in future therapeutic strategies. NK cells are cytotoxic against malignant cells in leukemia, thereby mounting a defense against tumor cells. In leukemic patients, NK cells decline as the disease progresses from the leukemia blast crisis. Also, NK cells isolated from patients with CML exhibit reduced cytotoxicity. Since NK cells exert cellular cytotoxicity and produce an adaptive immune response following the release of various cytokines, they serve an important role in the antitumor immune response (36). Thus, the anti-leukemic function of NK cells is a novel immunotherapeutic approach for CML.

\section{Therapeutic targets in CML by NK cells}

NK cells exert cytotoxic activity against tumor cells and malignant cells in leukemia. Previous studies have reported that the number and function of NK cells decreases in leukemic patients. The anti-leukemic action of NK cells is relatively low-level in patients with CML (62-64). However, a previous study demonstrated that $\mathrm{T}$ cells and NK cells are observed in patients as a consequence of receiving drug treatment (65).

The NKG2D NK cell receptor is activated by recognizing its ligands (NKG2DLs) on malignant cells, provoking the cytotoxic action of NK cells. The majority of tumor cells express NKG2DLs, including MICA, MICB, ULBP1 and ULBP2. MICA ligand is expressed on all leukemia cells and is associated with the immune response and anti-tumor activity. Thus, it is indicated that the NKG2D/MICA interaction has an important role in NK cell cytotoxicity against leukemia (62).

NK cells further influence the treatment of CML when patients with CML are difficult to treat with TKIs. In hematopoietic stem cell transplantation (HSCT), NK cell alloreactivity diminishes the rate of leukemia relapse and protects against an immunological GVH effect. This process is mediated by the 'missing-self' recognition on NK cells, whereas this phenomenon remains incomplete $(66,67)$. Therefore, it is necessary to investigate this process in order to develop an NK cell therapy for $\mathrm{CML}$ in the future.

\section{NK cell alloreactivity controls the GVH effect}

HSCT is also efficacious for myeloid malignancies, which require high doses of chemotherapy $(68,69)$. HSCT exerts its influence by inducing an immunological GVH effect. NK cells are thought to contribute to the GVH effect without directly causing graft-versus-host disease (GVHD) (70). NK cell alloreactivity regulated by their receptors is increased in the absence of HLA ligands. The missing-self represents an inhibitory effect of NK cells, which responds to activating signals to trigger the lysis of tumor targets. In addition, NK cells work in combination with the adaptive immune response to aggravate GVHD $(70,71)$.

\section{Adoptive NK cell therapy in hematopoiesis}

In hematopoietic transplants, donors having a KIR-ligand mismatch may be selected, thereby creating an environment in which recipient tumor cells act against alloreactive donor NK cells due to the missing KIR ligand (67,70). The alloreactivity of NK cells relies on the expression of a KIR and its MHC-I ligand in the donor, and the absence of the MHC-I ligand in the recipient. KIR and MHC-I ligand matching displays are summarized in Fig. 5. There are two principal donor-recipient mismatch models. The first mismatch model comprises donor and recipient KIR ligands (ligand-ligand model), and the second model includes recipients having no expression of KIR ligands for donor-inhibitory KIR receptors (missing self-model) (70). In alloreactive NK-cell responses, KIR2DS1 expression offers a striking advantage since it enables the efficient killing of $\mathrm{C} 2 / \mathrm{C} 2$ or $\mathrm{C} 1 / \mathrm{C} 2$ myelomonocytic dendritic cells and T-cell blasts. The interaction between KIR3DS1 and human leukocyte antigen (HLA)-Bw4 leads to a prognosis of slow disease progression following autologous stem cell transplantation (SCT) in patients with multiple myeloma (71-74). Thus, KIR2DS1 and KIR3DS1 expression in donor NK cells considerably increases the efficacy of the alloreactive NK-cell subset. A previous study reported a higher risk of developing CML due to the prevalence of tolerogenic NK cells, resulting from an increase in inhibitory signals generated by binding of iKIRs to ligands in the homozygous state. Other studies have reported a notable response to first or second generation TKI therapy to increase NK cell activity, mediated at least in part by aKIRs or a mismatch between iKIRs and their respective ligands $(74,75)$. The reason why the presence of active NK cells is paradoxically associated with a disadvantageous therapeutic response in CML remain poorly understood. One study undertook to further investigate the role of KIR receptors and their ligands at the gene level of a homogeneous group of CML patients and controls $(76,77)$. It was reported that KIR-HLA mismatches had a negative impact on the disease course of CML, and that the KIR-ligand combination KIR2DS2/KIR2DL2-absent/HLA-C1-present was markedly reduced in patients with CML $(76,77)$.

\section{KIR genes in patients with leukemia}

Another study analyzed the KIR genotype in patients with hematological diseases (35). Sequence specific primer-polymerase chain reaction (PCR-SSP) analysis was performed for the amplification of six inhibitory $K I R$ genes (KIR2DL1-2DL4 and 3DL1-3DL2) and six activating KIR genes (KIR2DS1-S5 and 3DS1). The PCR-SSP analysis determined the KIR genotypes of 54 patients with leukemia, including patients with AML, ALL, CML myelodysplastic syndrome and acute myeloid-lymphoblast leukemia. The results of the present study demonstrated that the frequency of activation of the KIR genes 2DS1, 3DS1 and 2DS3 was increased in standard-risk patients with acute AML compared with high-risk patients with acute AML, but there was no association with CML (35). 


\section{Conclusions}

This review has determined that understanding of NK cell functions and signals may improve the treatment of tumors, particularly in CML. Previous and current CML therapeutic strategies were summarized, from TKI treatment to immunotherapy.

Future clinical trials ought to consider that matching or mismatching of KIRs and KIR-ligands (HLAs) may be important for the treatment of CML using immunotherapy. Further research is required to investigate the cellular functions of specific KIR genes in patients with CML, and these studies may be applied to the improvement of therapeutic responses in CML.

\section{Acknowledgements}

The authors would like to thank the members of Professor Kwang-Hyun Baek's laboratory for their critical comments on the manuscript.

\section{Funding}

This study was supported by a grant from the Brain Korea 21 (BK21) PLUS project in Korea (grant no. 22A20130012771).

\section{Availability of data and materials}

Not applicable.

\section{Authors' contributions}

HRL and KHB performed the literature search and wrote the paper. KHB approved the final version of the manuscript to be published. All authors read and approved the manuscript and agree to be accountable for all aspects of the research in ensuring that the accuracy or integrity of any part of the work are appropriately investigated and resolved.

\section{Ethics approval and consent to participate}

Not applicable.

\section{Patient consent for publication}

Not applicable.

\section{Competing interests}

The authors declare that they have no competing interests.

\section{References}

1. Handgretinger R, Lang P and André MC: Exploitation of natural killer cells for the treatment of acute leukemia. Blood 127: 3341-3349, 2016.

2. Moretta L: Dissecting CD56 ${ }^{\mathrm{dim}}$ human NK cells. Blood 116: 3689-3691, 2010

3. Kannan GS, Aquino-Lopez A and Lee DA: Natural killer cells in malignant hematology: A primer for the non-immunologist. Blood Rev 31: 1-10, 2017.

4. Farag SS, Fehniger TA, Ruggeri L, Velardi A and Caligiuri MA Natural killer cell receptors: New biology and insights into the graft-versus-leukemia effect. Blood 100: 1935-1947, 2002.
5. Sun PD: Structure and function of natural-killer-cell receptors. Immunol Res 27: 539-548, 2003.

6. Willcox BE, Thomas LM and Bjorkman PJ: Crystal structure of HLA-A2 bound to LIR-1, a host and viral major histocompatibility complex receptor. Nat Immunol 4: 913-919, 2003.

7. Joyce MG, Tran P, Zhuravleva MA, Jaw J, Colonna M and Sun PD: Crystal structure of human natural cytotoxicity receptor NKp30 and identification of its ligand binding site. Proc Natl Acad Sci USA 108: 6223-6228, 2011.

8. Suck G, Linn YC and Tonn T: Natural killer cells for therapy of leukemia. Transfus Med Hemother 43: 89-95, 2016.

9. Parham P and Moffett A: Variable NK cell receptors and their MHC class I ligands in immunity, reproduction and human evolution. Nat Rev Immunol 13: 133-144, 2013.

10. Muntasel A, Ochoa MC, Cordeiro L, Berraondo P, López-Díaz de Cerio A, Cabo M, López-Botet M and Melero I: Targeting NK-cell checkpoints for cancer immunotherapy. Curr Opin Immunol 45: 73-81, 2017.

11. Lanier LL: Natural killer cell receptor signaling. Curr Opin Immunol 15: 308-314, 2003.

12. Sentman CL, Barber MA, Barber A and Zhang T: NK cell receptors as tools in cancer immunotherapy. Adv Cancer Res 95: 249-292, 2006.

13. Vivier E, Nune JA and Vély F: Natural killer cell signaling pathways. Science 306: 1517-1519, 2004.

14. Linnartz B, Wang Y and Neumann H: Microglial immunoreceptor tyrosine-based activation and inhibition motif signaling in neuroinflammation. Int J Alzheimers Dis 2010: 587463, 2010.

15. Pugh JL, Nemat-Gorgani N, Norman PJ, Guethlein LA and Parham P: Human NK cells downregulate Zap70 and Syk in response to prolonged activation or DNA damage. J Immunol 200: 1146-1158, 2018.

16. Teresa Rios-Paredes: Use of NK cells in haematological cancer therapy. Faculty of Medicine University of Oslo, 2014.

17. Verneris MR and Miller JS: KIR B or not to be?...that is the question for ALL. Blood 124: 2623-2624, 2014.

18. Gaafar A, Sheereen A, Almohareb F, Eldali A, Chaudhri N, Mohamed SY, Hanbali A, Shaheen M, Alfraih F, El Fakih R, et al: Prognostic role of KIR genes and HLA-C after hematopoietic stem cell transplantation in a patient cohort with acute myeloid leukemia from a consanguineous community. Bone Marrow Transplant 58: 1170-1179, 2018.

19. Long EO, Barber DF, Burshtyn DN, Faure M, Peterson M, Rajagopalan S, Renard V, Sandusky M, Stebbins CC, Wagtmann $\mathrm{N}$, et al: Inhibition of natural killer cell activation signals by killer cell immunoglobulin-like receptors (CD158). Immunol Rev 181: 223-233, 2001.

20. Ruggeri L, Capanni M, Urbani E, Perruccio K, Shlomchik WD, Tosti A, Posati S, Rogaia D, Frassoni F, Aversa F, et al: Effectiveness of donor natural killer cell alloreactivity in mismatched hematopoietic transplants. Science 295: 2097-2100, 2002.

21. Rajalingam R: Human diversity of killer cell immunoglobulin-like receptors and disease. Korean J Hematol 46: 216-228, 2011.

22. Fang F, Xiao W and Tian Z: NK cell-based immunotherapy for cancer. Semin Immunol 31: 37-54, 2017.

23. Ljunggren HG and Kärre K: In search of the missing self-MHC molecules and NK cell recognition. Immunol Today 11: 237-244, 1990.

24. Bottino C, Dondero A, Bellora F, Moretta L, Locatelli F, Pistoia V, Moretta A and Castriconi R: Natural killer cells and neuroblastoma: tumor recognition, escape mechanisms, and possible novel immunotherapeutic approaches. Front Immunol 5: 56, 2014.

25. Carotta S: Targeting NK cells for anticancer immunotherapy: Clinical and preclinical approaches. Front Immunol 7: 152, 2016.

26. Martinet L and Smyth MJ: Balancing natural killer cell activation through paired receptors. Nat Rev Immunol 15: 243-254, 2015

27. Shimasaki N, Coustan-Smith E, Kamiya T and Campana D: Expanded and armed natural killer cells for cancer treatment. Cytotherapy 18: 1422-1434, 2016.

28. Zhu Y, Huang B and Shi J: Fas ligand and lytic granule differentially control cytotoxic dynamics of natural killer cell against cancer target. Oncotarget 7: 47163-47172, 2016.

29. Childs RW and Carlsten M: Therapeutic approaches to enhance natural killer cell cytotoxicity against cancer: The force awakens. Nat Rev Drug Discov 14: 487-498, 2015.

30. Nückel H, Switala M, Sellmann L, Horn PA, Dürig J, Dührsen U, Küppers R, Grosse-Wilde H and Rebmann V: The prognostic significance of soluble NKG2D ligands in B-cell chronic lymphocytic leukemia. Leukemia 24: 1152-1159, 2010. 
31. Burger JA and Gribben JG: The microenvironment in chronic lymphocytic leukemia (CLL) and other B cell malignancies: Insight into disease biology and new targeted therapies. Semin Cancer Biol 24: 71-81, 2014.

32. Osman AE, AlJuryyan A, Alharthi $\mathrm{H}$ and Almoshary $\mathrm{M}$ : Association between the killer cell immunoglobulin-like receptor a haplotype and childhood acute lymphoblastic leukemia. Hum Immunol 78: 510-514, 2017.

33. Boissel N, Rea D, Tieng V, Dulphy N, Brun M, Cayuela JM, Rousselot P, Tamouza R, Le Bouteiller P, Mahon FX, et al: $\mathrm{BCR} / \mathrm{ABL}$ oncogene directly controls MHC class I chain-related molecule A expression in chronic myelogenous leukemia. J Immunol 176: 5108-5116, 2006.

34. Maru Y: Molecular biology of chronic myeloid leukemia. Cancer Sci 103: 1601-1610, 2012.

35. https://www.ncbi.nlm.nih.gov/books/NBK65916/

36. Danier AC, de Melo RP, Napimoga MH and Laguna-Abreu MT: The role of natural killer cells in chronic myeloid leukemia. Rev Bras Hematol Hemoter 33: 216-220, 2011.

37. Huang CH, Liao YJ, Fan TH, Chiou TJ, Lin YH and Twu YC: A Developed NK-92MI cell line with Siglec- $7^{\text {neg }}$ phenotype exhibits high and sustainable cytotoxicity against leukemia cells. Int J Mol Sci 19: E1073, 2018.

38. O'Brien SG, Guilhot F, Larson RA, Gathmann I, Baccarani M, Cervantes F, Cornelissen JJ, Fischer T, Hochhaus A, Hughes T, et al: Imatinib compared with interferon and low-dose cytarabine for newly diagnosed chronic-phase chronic myeloid leukemia. N Engl J Med 348: 994-1004, 2003.

39. Rossignol A, Levescot A, Jacomet F, Robin A, Basbous S, Giraud C, Roy L, Guilhot F, Turhan AG, Barra A, et al: Evidence for BCR-ABL-dependent dysfunctions of iNKT cells from chronic myeloid leukemia patients. Eur J Immunol 42: 1870-1875, 2012.

40. Heaney NB and Holyoake TL: Therapeutic targets in chronic myeloid leukaemia. Hematol Oncol 25: 66-75, 2007.

41. Pophali PA and Patnaik MM: The role of new tyrosine kinase inhibitors in chronic myeloid leukemia. Cancer J 22: 40-50, 2016

42. Shapira T, Pereg D and Lishner M: How I treat acute and chronic leukemia in pregnancy. Blood Rev 22: 247-259, 2008.

43. Goldman JM: How I treat chronic myeloid leukemia in the imatinib era. Blood 110: 2828-2837, 2007.

44. Garderet L, Santacruz R, Barbu V, van den Akker J, Carbonne B and Gorin NC: Two successful pregnancies in chronic myeloid leukemia patient treated with imatinib. Haematologica 92: e9-e10, 2007.

45. Roth MS and Foon KA: Alpha interferon in the treatment of hematologic malignancies. Am J Med 81: 871-882, 1986.

46. Giallongo C, La Cava P, Tibullo D, Parrinello N, Barbagallo I, Del Fabro V, Stagno F, Conticello C, Romano A, Chiarenza A, et al: Imatinib increases cytotoxicity of melphalan and their combination allows an efficient killing of chronic myeloid leukemia cells. Eur J Haematol 86: 216-225, 2011.

47. Millicovsky G, DeSesso JM, Kleinman LI and Clark KE: Effects of hydroxyurea on hemodynamics of pregnant rabbits: A maternally mediated mechanism of embryotoxicity. Am J Obstet Gynecol 140: 747-752, 1981.

48. Thauvin-Robinet C, Maingueneau C, Robert E, Elefant E, Guy H, Caillot D, Casasnovas RO, Douvier S and Nivelon-Chevallier A: Exposure to hydroxyurea during pregnancy: A case series. Leukemia 15: 1309-1311, 2001.

49. Yellu M, Pinkard S, Ghose A and Medlin S: CML in pregnancy: A case report using leukapheresis and literature review. Transfus Apher Sci 53: 289-292, 2015.

50. Ali R, Ozkalemkaş F, Ozkocaman V, Ozçelik T, Ozan U, Kimya Y and Tunali A: Successful pregnancy and delivery in a patient with chronic myelogenous leukemia (CML), and management of CML with leukapheresis during pregnancy: A case report and review of the literature. Jpn J Clin Oncol 34: 215-217, 2004.

51. Cayssials E and Guilhot F: Chronic myeloid leukemia: Immunobiology and novel immunotherapeutic approaches. BioDrugs 31: 143-149, 2017.

52. Mahoney KM, Rennert PD and Freeman GJ: Combination cancer immunotherapy and new immunomodulatory targets. Nat Rev Drug Discov 14: 561-584, 2015.

53. Mumprecht S, Schürch C, Schwaller J, Solenthaler M and Ochsenbein AF: Programmed death 1 signaling on chronic myeloid leukemia-specific T cells results in T-cell exhaustion and disease progression. Blood 114: 1528-1536, 2009.
54. Zhang B, Chu S, Agarwal P, Campbell VL, Hopcroft L, Jørgensen HG, Lin A, Gaal K, Holyoake TL and Bhatia R: Inhibition of interleukin-1 signaling enhances elimination of tyrosine kinase inhibitor-treated CML stem cells. Blood 128: 2671-2682, 2016

55. Ågerstam H, Hansen N, von Palffy S, Sandén C, Reckzeh K, Karlsson C, Lilljebjörn H, Landberg N, Askmyr M, Högberg C, et al: IL1RAP antibodies block IL-1-induced expansion of candidate CML stem cells and mediate cell killing in xenograft models. Blood 128: 2683-2693, 2016.

56. Arranz L, Arriero MD and Villatoro A: Interleukin- $1 \beta$ as emerging therapeutic target in hematological malignancies and potentially in their complications. Blood Rev 31: 306-317, 2017.

57. Zhao K, Yuan S, Yin L, Xia J and Xu K: Potential efficacy of human IL-1RAP specific CAR-T cell in eliminating leukemic stem cells of chronic myeloid leukemia. J Leukemia 5: 232, 2017

58. Stramucci L and Perrotti D: Twisting IL-1 signaling to kill CML stem cells. Blood 128: 2592-2593, 2016

59. Tarafdar A, Hopcroft LE, Gallipoli P, Pellicano F, Cassels J, Hair A, Korfi K, Jørgensen HG, Vetrie D, Holyoake TL, et al: CML cells actively evade host immune surveillance through cytokine-mediated downregulation of MHCII expression. Blood 129: 199-208, 2017.

60. Levescot A, Flamant S, Basbous S, Jacomet F, Féraud O, Anne Bourgeois E, Bonnet ML, Giraud C, Roy L, Barra A, et al: BCR-ABL-induced deregulation of the IL-33/ST2 pathway in $\mathrm{CD} 34^{+}$progenitors from chronic myeloid leukemia patients. Cancer Res 74: 2669-2676, 2014.

61. Rocca S, Carrà G, Poggio $\mathrm{P}$, Morotti $\mathrm{A}$ and Brancaccio $\mathrm{M}$ : Targeting few to help hundreds: JAK, MAPK and ROCK pathways as druggable targets in atypical chronic myeloid leukemia. Mol Cancer 17: 40, 2018.

62. Pierson BA and Miller JS: The role of autologous natural killer cells in chronic myelogenous leukemia. Leuk Lymphoma 27: 387-399, 1997.

63. Verfaillie C, Kay N, Miller W and McGlave P: Diminished A-LAK cytotoxicity and proliferation accompany disease progression in chronic myelogenous leukemia. Blood 76: 401-408, 1990.

64. Pawelec G, Schneider E, Ehninger G, Rehbein A and Schmidt H: Partial correction of defective generation of lymphokine-activated killer cells in patients with chronic myelogenous leukaemia after in vivo treatment with interferon-alpha (Wellferon). Cancer Immunol Immunother 29: 63-66, 1989.

65. Mustjoki S, Ekblom M, Arstila TP, Dybedal I, EplingBurnette PK, Guilhot F, Hjorth-Hansen H, Höglund M, Kovanen P, Laurinolli T, et al: Clonal expansion of T/NK-cells during tyrosine kinase inhibitor dasatinib therapy. Leukemia 23: 1398-1405, 2009.

66. Kijima M, Gardiol N and Held W: Natural killer cell mediated missing-self recognition can protect mice from primary chronic myeloid leukemia in vivo. PLoS One 6: e27639, 2011.

67. Zhao XY, Chang YJ, Xu LP, Zhang XH, Liu KY, Li D and Huang XJ: HLA and KIR genotyping correlates with relapse after T-cell-replete haploidentical transplantation in chronic myeloid leukaemia patients. Br J Cancer 111: 1080-1088, 2014.

68. Necchi A, Lanza F, Rosti G, Martino M, Farè E, Pedrazzoli P and European Society for Blood and Marrow Transplantation, Solid Tumors Working Party (EBMT-STWP) and the Italian Germ Cell Cancer Group (IGG): High-dose chemotherapy for germ cell tumors: Do we have a model? Expert Opin Biol Ther 15: 33-44, 2015.

69. Zahid U, Akbar F, Amaraneni A, Husnain M, Chan O, Riaz IB, McBride A, Iftikhar A and Anwer F: A review of autologous stem cell transplantation in lymphoma. Curr Hematol Malig Rep 12: 217-226, 2017.

70. Kim S, Poursine-Laurent J, Truscott SM, Lybarger L, Song YJ, Yang L, French AR, Sunwoo JB, Lemieux S, Hansen TH, et al: Licensing of natural killer cells by host major histocompatibility complex class I molecules. Nature 436: 709-713, 2005.

71. Sivori S, Carlomagno S, Falco M, Romeo E, Moretta L and Moretta A: Natural killer cells expressing the KIR2DS1-activating receptor efficiently kill T-cell blasts and dendritic cells: Implications in haploidentical HSCT. Blood 117: 4284-4292, 2011. 
72. Gabriel IH, Sergeant R, Szydlo R, Apperley JF, DeLavallade H, Alsuliman A, Khoder A, Marin D, Kanfer E, Cooper N, et al: Interaction between KIR3DSI and $H L A-B w 4$ predicts for progression-free survival after autologous stem cell transplantation in patients with multiple myeloma. Blood 116: 2033-2039, 2010.

73. Middleton D, Diler AS, Meenagh A, Sleator C and Gourraud PA: Killer immunoglobulin-like receptors (KIR2DL2 and/or KIR2DS2) in presence of their ligand (HLA-C1 group) protect against chronic myeloid leukaemia. Tissue Antigens 73: 553-560, 2009.

74. Marin D, Gabriel IH, Ahmad S, Foroni L, de Lavallade H, Clark R, O'Brien S, Sergeant R, Hedgley C, Milojkovic D, et al: KIR2DS1 genotype predicts for complete cytogenetic response and survival in newly diagnosed chronic myeloid leukemia patients treated with imatinib. Leukemia 26: 296-302, 2012.
75. Kreutzman A, Jaatinen T, Greco D, Vakkila E, Richter J Ekblom M, Hjorth-Hansen H, Stenke L, Melo T, Paquette R, et al: Killer-cell immunoglobulin-like receptor gene profile predicts good molecular response to dasatinib therapy in chronic myeloid leukemia. Exp Hematol 40: 906-913, 2012.

76. La Nasa G, Caocci G, Littera R, Atzeni S, Vacca A, Mulas O, Langiu M, Greco M, Orrù S, Orrù N, et al: Homozygosity for killer immunoglobin-like receptor haplotype A predicts complete molecular response to treatment with tyrosine kinase inhibitors in chronic myeloid leukemia patients. Exp Hematol 41: 424-431, 2013.

77. Zhao XY, Chang YJ and Huang XJ: Differential expression levels of killer immunoglobin-like receptor genotype in patients with hematological malignancies between high-risk and standard-risk groups. Zhongguo Shi Yan Xue Ye Xue Za Zhi 16: 746-749, 2008 (In Chinese). 